\title{
La organización turística del territorio en la frontera
}

DOI: 10.22403/UQROOMX/TYP08/01

\author{
Daniel Blasco Franch \\ Jaume Guia Julve \\ Lluís Prats Planagumà \\ Universitat de Girona
}

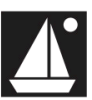

\section{Resumen}

En la actualidad, existen diversos debates académicos generados alrededor de los destinos turísticos, como I) la propensión o sensibilidad del turista frente a recorrer distancias en función de la motivación por visitar un determinado atractivo (Di Matteo, y Di Matteo 1996: 103);2) la creciente importancia de la gestión territorial de las marcas, de sus recursos turísticos (potenciales y desarrollados) y de su coherente distribución turísticamente hablando, en el territorio (Blasco Franch, Guia Julvie y Prats Planagumd, 2009 y 2010); 3) la influencia de los aspectos históricos y sociológicos en la creación de una identidad turística colectiva en nuevos destinos turísticos (Palmer, 1999: 313); o, en definitiva, 4) la desconexión entre muchos productos de los mercados actuales y la demanda (Urry, 1990 y 200I). Todos estos debates convergen en una misma idea: la importancia de la concepción del espacio psicológico y real por parte del turista por un lado y de los organizadores de destinos por el otro, y su determinante influencia en el desarrollo turístico de las regiones.

En el caso de los territorios situados en la frontera, resulta fundamental para comprender su evolución. Las distancias en la frontera son especialmente etéreas. Por una parte, los territorios en la frontera son siempre,por definición, periféricos del centro,por lo que a menudo se encuentran "alejados" de las políticas y acciones de los organismos 
de gestión de los destinos y demás instituciones competentes con concepciones centralistas (Lovelock y Boyd, 2006: 143). Así pues, existen potencialidades derivadas de las interdependencias inter e intradestinaciones en la frontera, que están siendo desaprovechadas debido a que la planificación y consumo turístico del territorio se hace siguiendo criterios administrativos, y no siempre se logra el desarrollo óptimo. Por otra parte, la (no) permeabilidad de la frontera también puede facilitar (o dificultar) enormemente el correcto desarrollo de la actividad turística. Todos los productos pueden parecer mucho más cercanos o alejados en función de las características de la frontera que los separe (Zhang, Inbakaran y Jackson, 2006: 182).

Palabras Clústeres turísticos, análisis espacial, microrregiones

CLAVE turísticas, planificación territorial del turismo, turismo transfronterizo 


\section{Introducción}

La mayor parte de la literatura sobre organización de destinos turísticos toma como base la oferta de productos existentes en su territorio exclusivamente: ¿qué tengo en mi territorio? (Michael, 2007 y 2003: I33; Jackson y Murphy, 2006: I 0 I8; Novelli, 2005: I I4 I; Porter, 2003:545). Por el contrario, en muchas menos ocasiones se encuentran ejemplos que partan de la mirada del turista a nuestro territorio (según el turista iqué es -y qué puede ser- mi territorio turísticamente?). De tal suerte, existe un gap en la literatura de gestión de los destinos turísticos en lo relativo a las oportunidades desaprovechadas derivadas de nuevas miradas, interpretaciones o "versiones" del territorio. Al fin y al cabo, los turistas consumen el territorio debido a los productos que hay en él, pero generalmente lo que en realidad les interesa son los productos en sí mismos. La cuestión está en saber si los responsables de destinos pueden concebir estrategias para el desarrollo de los destinos turísticos que permeabilicen las estructuras administrativas actuales y prioricen criterios de empresa, de producto, $y$ de mercado o demanda, que garanticen la máxima competitividad y sostenibilidad de las propuestas turísticas resultantes (Jansen-Verbeke y Lievois, 2002; Weidenfeld, Butler y Williams, 2010: 2).

Así pues, ¿cómo debemos regionalizar turísticamente el territorio de forma que se aproveche el mayor número de oportunidades? El presente artículo tiene por objetivo revisar algunas teorías sobre el desarrollo turístico de los territorios, poniendo énfasis en la importancia de las distancias entre los productos y en una concepción fundada en la perspectiva de la demanda. Por medio de la metodología de cluster analysis, la propuesta consiste en la categorización y agrupación temática de los productos turísticos cercanos existentes en el territorio con base en determinados criterios especialmente sensibles para los turistas (por ejemplo la existencia de productos sinérgicos cercanos o de áreas temáticas), con la finalidad de proyectar nuevas concepciones del territorio en clústeres o aglomeraciones territoriales distintas.

En conclusión, se plantea un modelo de análisis espacial que muestra nuevas interpretaciones o lecturas de la realidad, más justas, equilibradas y optimizadas del territorio, con la intención de hacer aflorar o emerger aquellas propuestas o combinaciones entre los recursos y atractivos turísticos que más se acerquen a las necesidades de los turistas actuales, independientemente de la existencia de fronteras existentes intra o inter estos recursos o atractivos. 
Si bien la propuesta es exportable a cualquier territorio, a modo de ejemplo se presenta el caso de la Cerdanya, territorio situado en la frontera españolafrancesa, dividida geográficamente por una cadena montañosa (los Pirineos).

\section{Marco teórico: los procesos de clusterización en territorios fronterizos}

El contexto actual de aumento progresivo y constante de la complejidad de los ecosistemas empresariales, el incremento de las exigencias de los mercados, así como los azotes de las crisis económico-financieras, son las principales razones por las que las empresas se deberán preparar para afrontar escenarios de futuro inciertos para la actividad turística, sobre todo en aquellos destinos más maduros. Además, en el contexto de análisis crítico de los actuales destinos turísticos, es fundamental adquirir un profundo conocimiento sobre la propensión o resistencia hacia la clusterización de las empresas que constituyen los destinos turísticos. Finalmente, el componente transfronterizo de algunos destinos también confiere unas características territoriales muy especiales al territorio.

\section{La construcción de nuevos destinos turísticos}

En el futuro, muchas empresas se verán obligadas a reforzar determinadas relaciones empresariales, en las que coexistan la competencia y la cooperación. Es precisamente la naturaleza dual de estas relaciones la que estimula el ecosistema empresarial hasta hacerlo altamente competitivo en el nivel global, y genera así mayores beneficios para casi todos los integrantes del ecosistema o clúster (Krugman, 1994; Kaufman y Rousseeuw, 2005).

Los clústeres, ya sean territoriales, temáticos/especializados, etc., han sido un fenómeno tratado con amplitud en la literatura académica, sobre todo con respecto a la dimensión de la oferta, fundamentalmente a partir de aproximaciones macroeconómicas (Porter, 1998;2003:546), pero no de forma tan extensa en relación con la dimensión de la demanda. En cuanto a esta última, la variedad y concentración de la oferta juegan un papel primordial en el proceso de creación y consolidación de un destino.

Diversos estudios recientes analizan la creación de clústeres en el sector turístico (Tinsley y Lynch, 200 I: 367; Michael, 2003: I 33; y 2007; Nordin, 2003; 
Saxena, 2005: 277; Hall, 2005: I49; Novelli, Schmitz y Spencer, 2006: I I4I;Anton et al., 2006). En cualquier caso, se constata en la mayoría de los artículos publicados hasta el momento, que se parte de la base territorial de las marcas o destinos turísticos preexistentes (Poon, 1993; Michael, 2003: I33). Por ello, no se considera la posibilidad de hacer aflorar nuevas realidades turísticas derivadas de la reinterpretación del territorio con nuevos criterios de la base territorial. Además, algunos esfuerzos encaminados a resolver esta cuestión (Anton et al., 2006) todavía resultan excesivamente parciales.

\section{El turismo en la frontera}

El turismo no entiende de fronteras, de hecho más bien representa su antítesis: si bien las fronteras sirven básicamente para impermeabilizar territorios, el turismo es, en esencia, el acto de cruzarlas. Incluso algunas fronteras resultan ser atractivos turísticos por sí mismas (Timothy, 1995: 525). Existen diversos ejemplos en la literatura académica de desarrollo turístico en regiones transfronterizas que se han llevado a cabo de forma exitosa, como el caso de la región de Tornio, entre Suecia y Finlandia (Prokkola, 2007: I20); el de la frontera entre Canadá y Estados Unidos (Di Matteo y Di Matteo, 1996: I03); o entre los seis países a orillas del río Mekong (Sofield, 2006: I02). En cualquier caso, las relaciones turísticas transfronterizas no están exentas de problemas, tanto en el plano estatal (Loannides, Nielsen y Billing, 2006: 122), como en el regional y el local (Lovelock y Boyd, 2006: 143). Además, a menudo, las estructuras turísticas preexistentes en ambos lados de la frontera resultan un obstáculo para el desarrollo de nuevos destinos turísticos transfronterizos (Loannides, Nielsen y Billing, 2006: I38). Otra dificultad se encuentra en las diferencias en la percepción de la frontera por parte de los turistas y de los residentes. Por ejemplo, para los agentes de destinos fronterizos, ésta se percibe como una barrera para el desarrollo, mientras que, para los turistas, con frecuencia representa una contribución positiva para la experiencia (Lovelock y Boyd, 2006: 145). Por ello, generalmente la personalidad de muchas regiones fronterizas es dual: por un lado, se trata de un territorio con un enorme volumen de movimientos, contactos y relaciones; por el otro, la frontera actúa como barrera más o menos rígida entre los territorios colindantes. Por tanto, los obstáculos y conflictos de intereses entre los países vecinos y las regiones transfronterizas pueden minar los beneficios potenciales derivados de la colaboración transfronteriza. 
Al fin y al cabo, según Gilbert (1960: I57), las “regiones son como individuos, tienen caracteres muy distintos y éstos se desarrollan y cambian constantemente". De hecho, el destino turístico es una construcción socioespacial caracterizada por una idea de región construida socialmente, que va más allá de los aspectos físicos o administrativos (Giddens, 1985). Así pues, los destinos se pueden entender como productos en constante cambio, derivados de una cierta combinación de aspectos sociales, políticos y económicos que son específicos en el espacio y tiempo (Getz, 1999: 23).

\section{Metodología}

El presente estudio tiene por objetivo analizar la presencia y disposición de los productos turísticos en el territorio con el fin de obtener conclusiones sobre la interacción que dicha oferta puede obtener con la demanda turística. El territorio que se ha examinado es el de la frontera entre España y Francia, en la región de Cataluña.

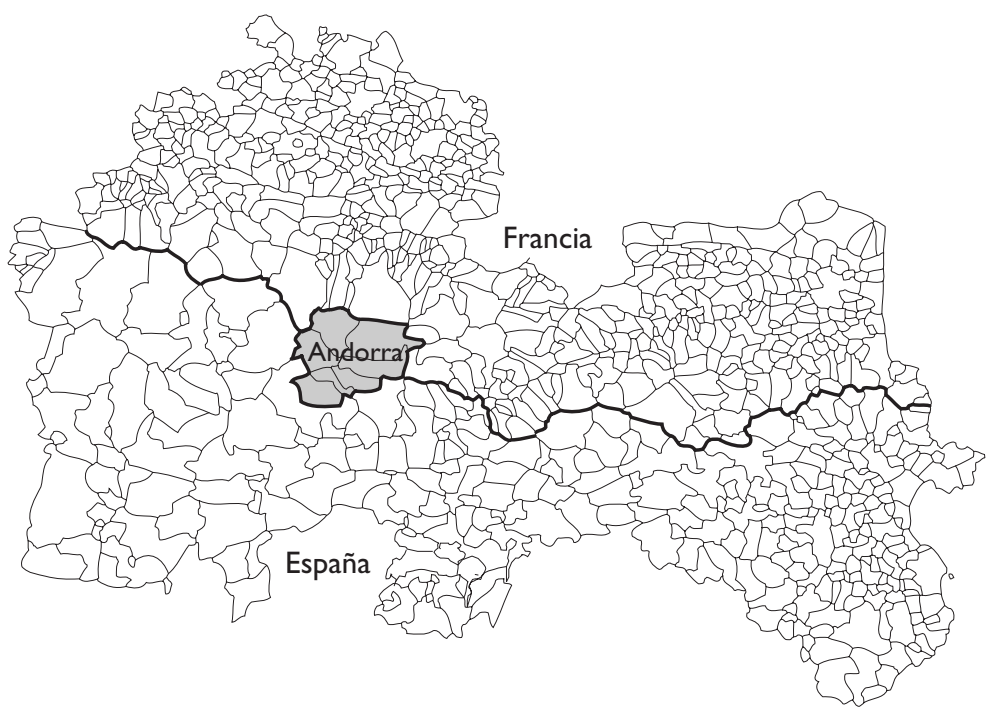

Figura I.Territorio en La frontera entre España y Francia 
Para desarrollar esta investigación, se ha seguido el proceso que se detalla a continuación.

I.Para empezar, se ha hecho una matriz con las distancias entre los municipios de la región de la Cataluña norte y sur, en la frontera entre España y Francia, con un total de 859 municipios (véase figura I). La distancia máxima dentro del territorio transfronterizo analizado es de $430 \mathrm{~km}$.

2. A continuación, se ha llevado a cabo un análisis clúster para determinar qué territorios cuentan con unas distancias internas suficientemente compactas para ser consideradas un clúster desde la perspectiva del consumo turístico. En concreto, se han agrupado los 859 municipios del territorio transfronterizo en un abanico que va de los dos a los 50 clústeres.

3. Se ha observado un comportamiento muy compacto en 49 municipios del territorio de la Cerdanya, dividido por la frontera, con 17 municipios del lado español y 32 del francés, lo que implica que estos municipios están más cercanos entre sí que respecto a aquellos del entorno nacional respectivo. Las distancias internas máximas del clúster identificado son de $75 \mathrm{~km}$ de largo por una distancia máxima inferior a los $40 \mathrm{~km}$ de ancho. La distancia entre el municipio más importante del clúster (Puigcerdà) y los municipios más alejados es de $4 \mathrm{I} \mathrm{km}$ hasta Réal, y de $35 \mathrm{~km}$ hasta Lles de Cerdanya.

4. Una vez identificado el territorio con una proximidad en términos de distancias en kilómetros con potencial para ser considerada una posible área para el desarrollo turístico, se ha construido una base de datos de productos turísticos, con un total de 863 en los 49 municipios del territorio transfronterizo de la Cerdanya, con el objetivo de identificar la presencia y acumulación de productos turísticos. Se identificaron ocho categorías de productos, y sus subcategorías pueden apreciarse en el cuadro I.

5. Se ha elaborado una representación gráfica por medio de la proyección de mapas territoriales temáticos, tras haber identificado los ejes temáticos de desarrollo turístico con mayor potencial.

En resumen, este proceso metodológico debe permitir obtener información sobre la situación actual de equilibrio/descompensación de productos en determinadas áreas del territorio, así como avanzar en la identificación de nuevas propuestas con potencial para el desarrollo turístico en la frontera. 
La organización turística

del territorio en la frontera

Cuadro I. Categorías y subcategorías de productos turísticos

\begin{tabular}{ll}
\hline Categoría (8) & \multicolumn{1}{c}{ Subcategorías (2I) } \\
\hline Cultura & Arquitectura; museos; arqueología; artesanía; eventos \\
\hline Activo & Aventura; deporte; golf; motor; nieve; salidas; eventos \\
\hline Esquí & Alojamiento; nieve; eventos \\
\hline Naturaleza & Accidentes geográficos; espacio natural \\
\hline Rutas & Senderismo; cultura; naturaleza \\
\hline Ocio & Compras \\
\hline Salud-Belleza & Alojamiento \\
\hline Infraestructura & Alojamiento; restauración; transporte; ocio \\
\hline
\end{tabular}

\section{Resultados}

A continuación se ofrecen los resultados derivados de la aplicación del proceso de clusterización de los municipios del territorio de la Cerdanya, entre España y Francia, con base en los productos turísticos existentes en tales municipios.

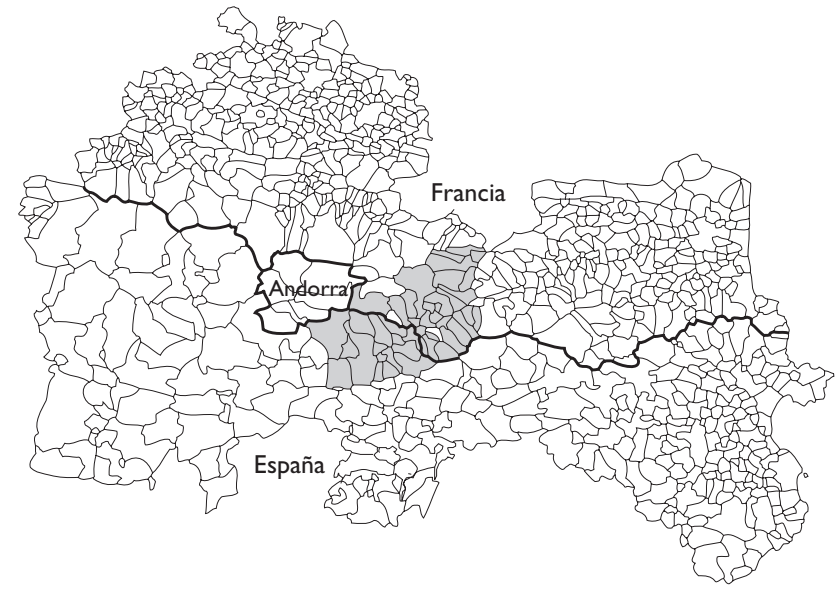

FIGURA 2. MUNICIPIOS FRONTERIZOS CON PRODUCTOS DE TURISMO CULTURAL 
Respecto al rubro "Cultura", se han identificado 222 productos, 8 I en el lado español y 14 I en el francés. Se trata de la categoría con una presencia más masiva de productos, sobre todo los relacionados con la arquitectura (I43 productos presentes en 14 municipios españoles y 27 franceses) y con los eventos ( 53 productos presentes en 14 municipios españoles y 18 franceses).

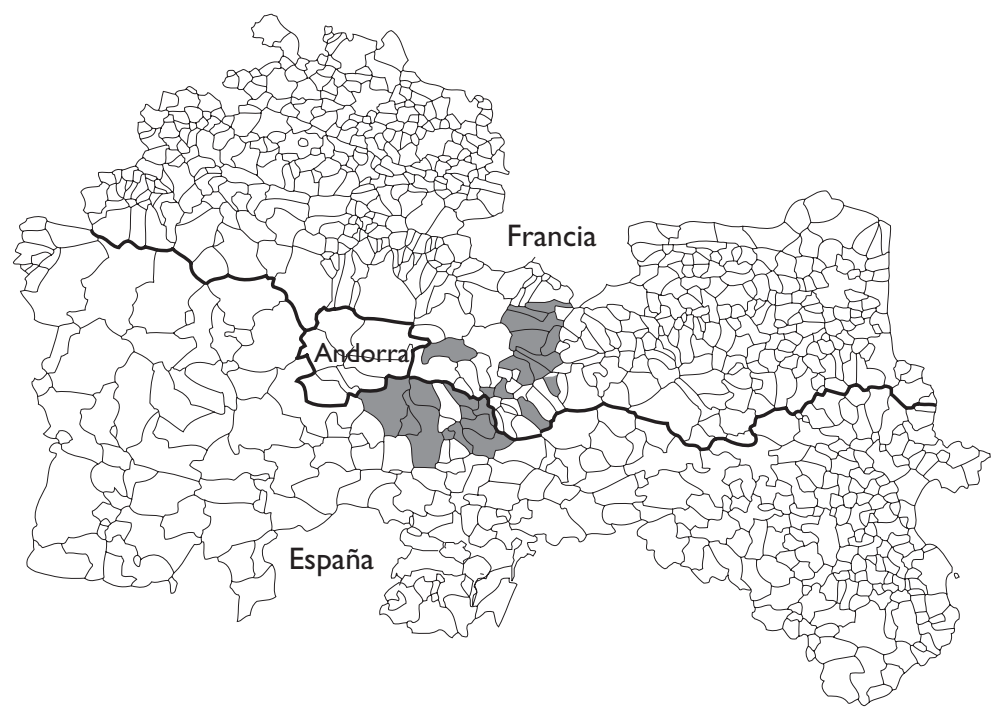

FIGURA 3. MUNICIPIOS FRONTERIZOS CON PRODUCTOS DE TURISMO ACTIVO

También se han identificado 76 productos de turismo activo, 58 en el lado español y 18 en el francés. Se considera el segundo eje temático del territorio después del cultural, con un reparto bastante generalizado de los productos entre las distintas subcategorías, predominando la de turismo de nieve (con 14 municipios y 21 productos -ocho en España y 13 en Francia-) y de aventura (ocho municipios y 22 productos - 19 en España y tres en Francia-).

Al observar la gran cantidad de productos vinculados con la actividad del esquí (3I), se ha creado una categoría específica para su análisis. Los resultados permiten afirmar que el esquí se postula como el tercer gran eje temático del territorio, con presencia en 17 municipios: en Francia hay 15 productos en II municipios y en España 16 productos en seis municipios. 
La organización turística

del territorio en la frontera

$\Delta_{18}$

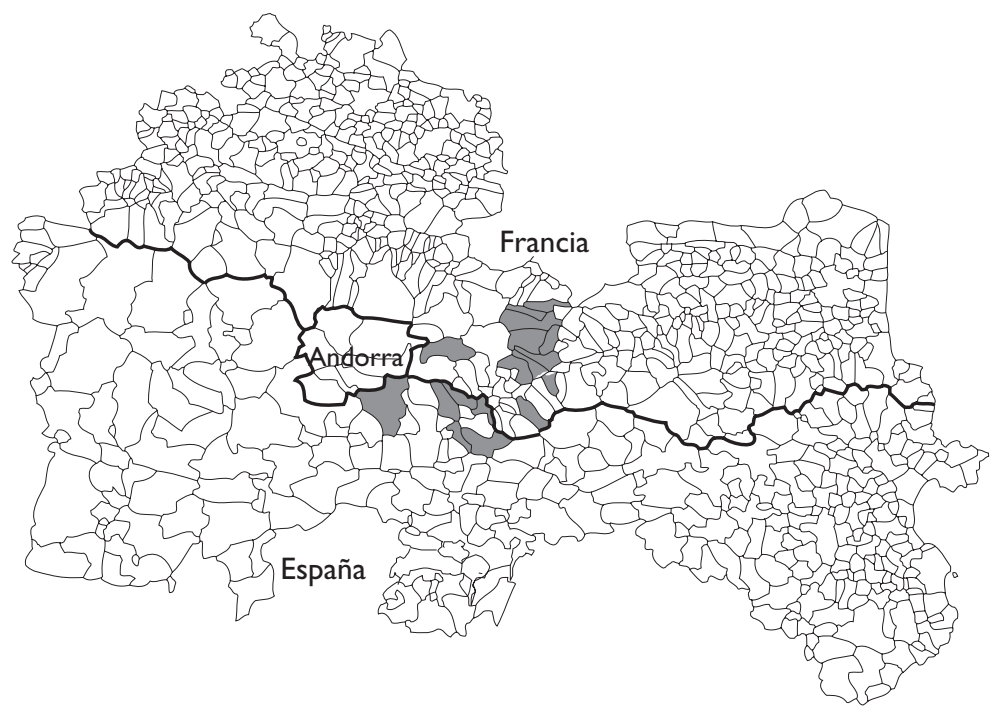

FIGURA 4. MUNICIPIOS FRONTERIZOS CON PRODUCTOS DE TURISMO DE ESQUÍ

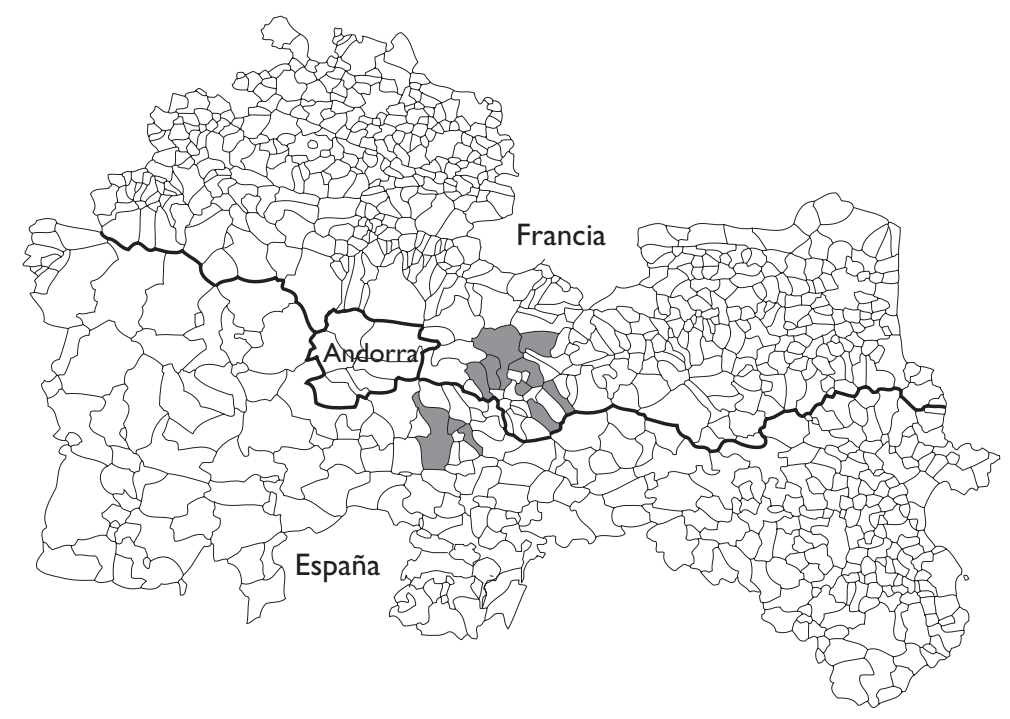

FIGURA 5. MUNICIPIOS FRONTERIZOS CON PRODUCTOS DE TURISMO DE NATURALEZA 
Daniel Blasco Franch

Jaume Guia Julve

Lluís Prats Planagumà

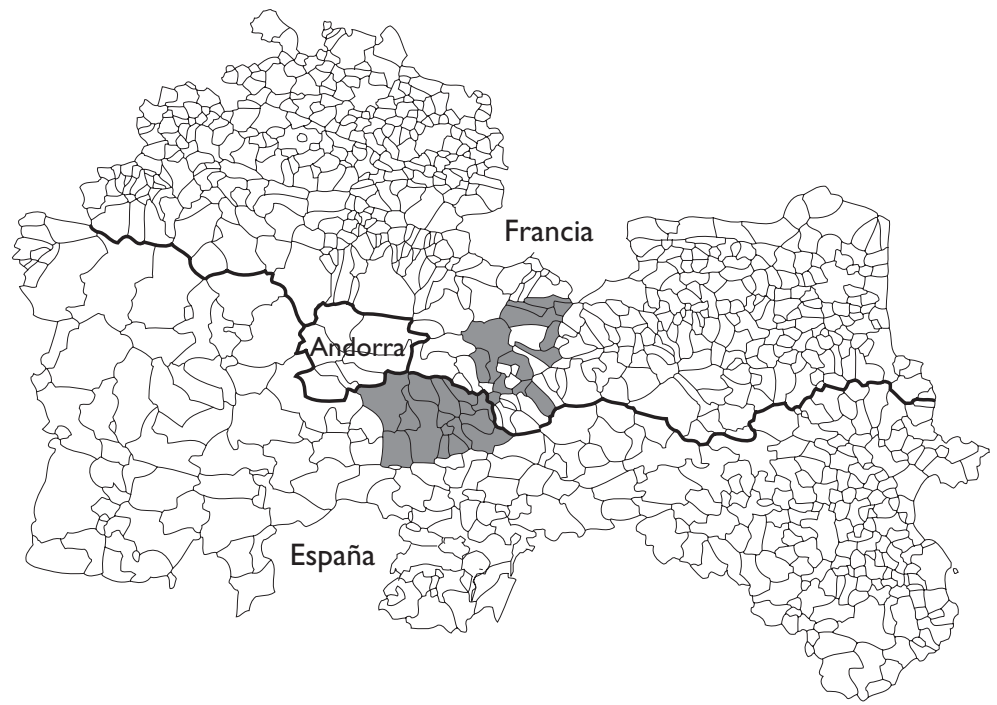

FIGURA 6. MUNICIPIOS FRONTERIZOS CON PRODUCTOS DE INFRAESTRUCTURA HOTELERA

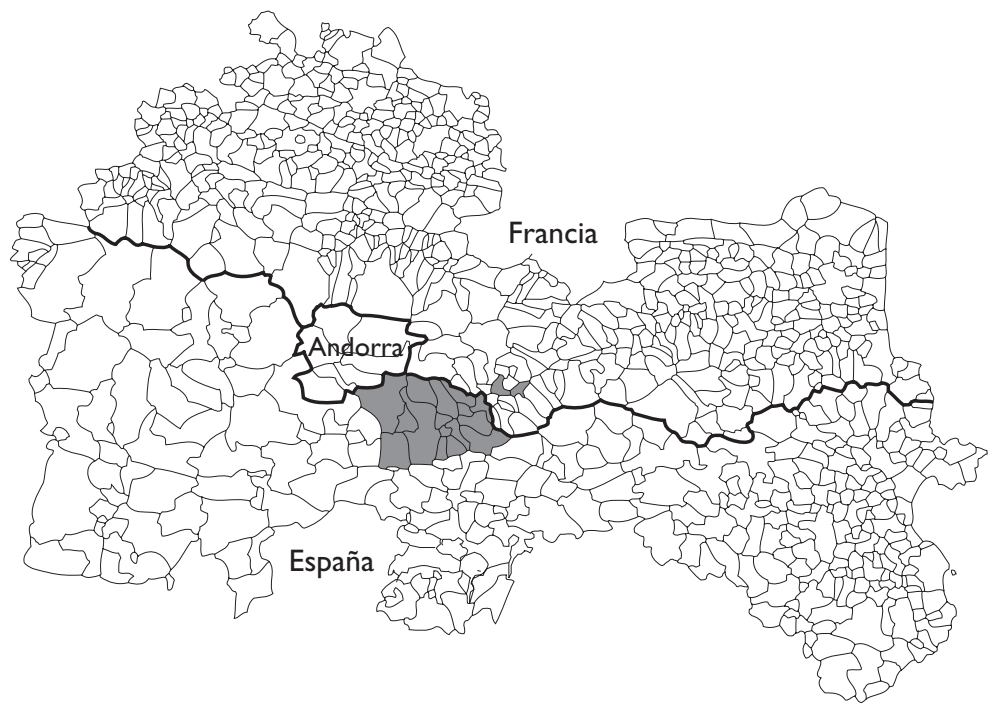

FIGURA 7. MUNICIPIOS FRONTERIZOS CON PRODUCTOS DE INFRAESTRUCTURA DE RESTAURACIÓN 
La última temática con un lugar relevante es la de "Naturaleza", con 38 productos repartidos en tres municipios españoles y ocho franceses.

Finalmente, en el ámbito de la infraestructura turística se han identificado 494 productos repartidos en 17 municipios españoles y 12 franceses. La subcategoría de alojamiento incluye 262 productos, y la gran mayoría de municipios cuenta con ellos, si bien hay una presencia muy superior en España con I36 productos, contra 126 en Francia.

Respecto a la restauración, esta subcategoría reporta 227 productos. La figura 7 refleja de forma evidente la diferencia en el desarrollo turístico de ambos lados de la frontera: mientras que en el español todos los municipios tienen oferta de restauración, en el francés sólo un municipio dispone de ella.

\section{Conclusiones y futuras investigaciones}

Las principales conclusiones derivadas del análisis de los mapas se pueden resumir de la siguiente forma:

I. Se constatan tres categorías de producto o temática transversales, que se encuentran dispersas de manera bastante homogénea en muchos municipios de ambos lados de la frontera: el turismo cultural, el turismo activo y el de esquí. Si bien se trata de tipologías de turismo habituales en zonas montañosas como la analizada (los Pirineos), en este caso la Cerdanya cuenta además con una proximidad entre los productos que le confieren una ventaja competitiva en su comercialización, que debe permitir diferenciarlos de otros similares, ya sea en territorios cercanos de los Pirineos o en cualquier otra región de montaña con potencial turístico de Europa.

2. Se constata un cierto grado de especialización en el desarrollo de productos turísticos en cada lado de la frontera, de modo que si la parte española se caracteriza por disponer de más infraestructura y productos de turismo activo, la francesa tiene más productos de turismo cultural. Ello seguramente tiene lógica atendiendo al desarrollo turístico de Francia y España hasta la fecha. Así, la mayor expansión de la infraestructura en España se debe al menor coste que tiene en relación con Francia.También se observa una especialización temática: Francia se ha distinguido por un crecimiento más intenso del turismo cultural, mientras que España, 
de los productos de turismo activo en zonas de interior, en un esfuerzo por diversificar el clásico producto de sol y playa del litoral.

3. Se constata la disparidad en el nivel de desarrollo turístico de la Cerdanya francesa y española. De hecho, el lado español predomina en la presencia de productos de la mayoría de categorías y subcategorías, con el evidente caso de la restauración. Pero Francia tiene casi el doble de municipios en el clúster que España (32 contra 17). El desarrollo turístico en ambos lados de la frontera seguramente sería otro si este territorio fuera un país independiente, como la vecina Andorra. Por ello, según se ha explicado, la concepción del clúster como un único territorio confiere potencialidades derivadas de la distinta especialización del territorio en cada parte de la frontera, sumando en conjunto una buena cantidad de sinergias como resultado final.

En conclusión, los resultados expuestos en este artículo representan un primer paso hacia el desarrollo de una metodología que permita realizar nuevas lecturas o interpretaciones del territorio, a partir de la existencia de productos turísticos y de la proximidad entre ellos. Para lograrlo, en investigaciones futuras se tendrán en cuenta otros factores como la cantidad y la importancia de los productos turísticos y atractivos presentes en el territorio, así como ahondar en su complementariedad y posibles efectos sinérgicos entre categorías y subcategorías, y el nivel o intensidad de interés que todo ello suscita en los turistas. No obstante, la propuesta iniciada en este artículo debería comportar una reflexión alrededor de la configuración de los destinos turísticos actuales, con el propósito de generar nuevas perspectivas de desarrollo territorial en el futuro.

\section{FUENTES CONSULTADAS}

Anton, S. et al. (2006). Clústers Turístics de Catalunya. Generalitat de Catalunya: Universitat de Girona/Universitat Rovira iVirgili (mimeo).

Blasco Franch, D., J. Guia Julve y LI. Prats Planagumà (2009). “Clústers de productos turísticos: el caso de Cataluña”. Dinámicas y Tendencias del Turismo Contemporáneo. Memoria del Primer Coloquio Internacional en la Cátedra Patrimonial en Turismo Sergio Molina. Ciudad Juárez:Universidad Autónoma de Ciudad Juárez. 
(2010). "Desarrollo de un clúster transfronterizo de enoturismo entre Francia y España”. Artículo aceptado en la revista Cahiers Européennes des Sciences Sociales. Université de Persignan Via Domitia. Di Matteo, L. y R. Di Matteo (1996). "An Analysis of Canadian Cross-Border Travel”. Annals of Tourism Research, 23 (I), I03-I 22.

Getz, D. (1999). "Resort-centred Tours and Development of the Rural Hinterland: the Case of Cairns and the Atherton Tablelands". The Journal of Tourism Studies, 10 (2), 23-34.

Giddens,A. (1985). “Time, Space and Regionalisation”, en D. Gregory y J. Urry (eds.). Social Relations and Spatial Structures. Nueva York: St. Martin's Press, 265-295.

Gilbert, E.W. (1960). “The Idea of the Region”. Geography, 45, I57-I 75. Hall, C.M. (2005). "Rural Wine and Food Tourism Cluster Network Development”, en D. Hall, I. Kirkpatrick y M. Mitchell (eds.). Rural

Tourism and Sustainable Business. Clevendon: Channel View, I49-I64. loannides, D., P. Nielsen y P. Billing (2006). "Transboundary Collaboration in Tourism: the Case of the Bothnian Arc". Tourism Geographies, 8 (2), I22-142.

Jackson, J.y P. Murphy (2006). "Clusters in Regional Tourism.An Australian Case”. Annals of Tourism Research, 33 (4), 1018-1035.

Jansen-Verbeke, M.y E. Lievois (2002).“Analysing Heritage Resources for Urban Tourism in European Cities”, en D.G. Pearce y R.W. Butler (eds.). Contemporary Issues in Tourism Development. Londres: Routledge, 8I - 108. Kaufman, L. y P.J. Rousseeuw (2005). Finding Groups in Data. An Introduction to Cluster Analysis. Nueva Jersey: John Wiley and Sons.

Krugman,P.(1994).'Location and Competition:Notes on Economic Geography”, en R.P. Rumelt, D.E. Schendel y D.J.Teece (eds.). Fundamental Issues in Strategy: A Research Agenda. Boston: Harvard Business School Press, 463-494.

Lovelock, B. y S. Boyd (2006). "Impediments to a Cross-Border Collaborative Model of Destination Management in the Catlins, New Zealand". Tourism Geographies, 8 (2), I43-I6I.

Michael, E.J. (2003).“Tourism Micro-clusters”. Tourism Economics, 9 (2), I 33- I 45. (2007). Micro-clusters and Networks: the Growth of Tourism, Advances in Tourism Research Series. Oxford: Elsevier. 
Nordin, S. (2003). Tourism Clustering and Innovation - Paths to Economic Growth and Development. Oestersund: European Tourism Research Institute, Mid-Sweden University.

Novelli, M., B. Schmitz y T. Spencer (2006).“Networks, Clusters and Innovation in Tourism:A UK Experience". Tourism Management, 27 (6), I I 4 I-I I 52.

Palmer, C. ( 1999). “Tourism and the Symbols of Identity". Tourism Management, 20, 313-32I.

Poon,A. (1993). Tourism, Technology and Competitive Strategies. Wallingford: CAB International.

Porter, M.E. (1998)."Clusters and Competition: New Agendas for Companies, Governments and Institutions", en M.E. Porter (ed.). On Competition. Boston: Harvard Business School Press, 197-287.

(2003). “The Economic Performance of Regions". Regional Studies, 37 (6), 545-546.

Prokkola, E.K.(2007).“Cross-border Regionalization and Tourism Development at the Swedish-Finnish Border:'Destination Arctic Circle'”. Scandinavian Journal of Hospitality and Tourism, 7 (2), I20-I38.

Saxena, G. (2005). "Relationships, Networks and the Learning Regions: Case Evidence from the Peak District National Park". Tourism Management, 26, 277-289.

Sofield, T. (2006). "Border Tourism and Border Communities: An Overview". Tourism Geographies, 8 (2), I02-I 2 I.

Timothy, D. (1995)."Political Boundaries and Tourism:Borders as TouristAttractions". Tourism Management, 16 (7), 525-532.

Tinsley, R.y P. Lynch (200I)."Small Tourism Business Networks and Destination Development". International Journal of Hospitality Management, 20, 367-378.

Urry,J. (1990). The Tourist Gaze. Leisure and Travel in Contemporary Societies. Newbury Park: Sage Publications.

(200 I). Globalizing the Tourist Gaze. Graz: Cityscapes Conference.

Weidenfeld, A., R. Butler y A.Williams (2010). "Clustering and Compatibility between Tourism Attractions". International Journal of Tourism Research, 12, I- 16.

Zhang, Z., R. Inbakaran y M.Jackson (2006).“Understanding Community Attitudes towards Tourism and Host-Guest Interaction in the Urban-Rural Border Region”. Tourism Geographies, 8 (2), 182-204. 